\title{
The Effects of Dexmedetomidine Continuous Rate Infusion (CRI) on Isoflurane Anaesthesia in Healthy Horses
}

\author{
Makram Sleiman, Dinko Dinev, Galina Simeonova \\ Department of Veterinary Surgery, Faculty of Veterinary Medicine, Trakia University, Stara Zagora, Bulgaria \\ Email: galinavet@abv.bg
}

Received 23 March 2016; accepted 31 May 2016; published 3 June 2016

Copyright (C) 2016 by authors and Scientific Research Publishing Inc.

This work is licensed under the Creative Commons Attribution International License (CC BY).

http://creativecommons.org/licenses/by/4.0/

(c) (;) Open Access

\begin{abstract}
The concept of the modern anaesthesiological technique partial intravenous anaesthesia (PIVA) is by means of infusion of different pharmacological agents as a supplement to inhalation anaesthetics to reduce the concentration of volatile agents maintaining surgical anaesthesia and to decrease their noxious side effects mainly on cardiovascular and respiratory systems. Alpha-2 agonists are agents with frequent use in equine practice either as sedatives or in general anaesthesia PIVA protocols. The most selective amongst them, dexmedetomidine, is characterized by fewer side effects, lower doses, and fast elimination which make it appropriate for application as a continuous rate infusion (CRI). The purpose of this study was to trace out the effects of dexmedetomidine continuous rate infusion (CRI) as part of PIVA using isoflurane on volatile agent requirements, cardiovascular function, respiration and coagulation parameters, and recovery in healthy horses. Six healthy horses with average age $9.0 \pm 5.1$ year and mean body weight $247.7 \pm 71.4 \mathrm{~kg}$ were subjected to either 3-hour lasting isoflurane or isoflurane-dexmedetomidine anaesthesia two weeks apart. The main clinical and anaesthesiological parameters were monitored in 10 minutes intervals. Electrolytes, acid-base, blood gases, and coagulation parameters were measured at the beginning and at the end of each anaesthesia. Recovery times and qualities were also recorded. The results showed that, the addition of dexmedetomidine by CRI at $1.75 \mu \mathrm{g} \cdot \mathrm{kg}^{-1} \cdot \mathrm{hour}^{-1}$ to isoflurane anaesthesia slightly reduced isoflurane requirement for maintenance of surgical anaesthesia but had negligible effects on the recovery time and quality. PIVA using dexmedetomidine and isoflurane produced respiratory acidosis similar to isoflurane anaesthesia alone but with significantly more pronounced hypoxaemia and hyperlactaemia. Both investigated anaesthesia protocols did not influence significantly haemocoagulation parameters.
\end{abstract}

\section{Keywords}

Isoflurane, Dexmedetomidine, Continuous Rate Infusion, Horses 


\section{Introduction}

Anaesthesia related mortality in horses is much higher than other species [1] [2] which makes anaesthetists to look after safer protocols for general anaesthesia. Equid size, behavior and physiology contribute to significant risks and challenges to inhalation anaesthesia in horses relative to other species.

Inhalation agents provide deep and long enough anaesthesia for surgical procedures but on the price of pronounced cardiorespiratory depression [3]. Isoflurane is the most widely used volatile agent in equine anaesthesia because of its potency, low blood solubility, and low price. As other inhalation anaesthetics, isoflurane causes dose- and time-dependent cardiovascular and respiratory depression. Infusion of sedative, analgesic, or anaesthetic drugs in addition to inhalation agent can provide a surgical plane of anaesthesia using reduced doses and therefore may decrease undesired side effects. This technique in called partial intravenous anaesthesia (PIVA) and several agents has been studied and proposed to be used by continuous rate infusion (CRI) to supplement volatile agents in horses [4] [5].

Alpha 2-adrenoceptor agonists are widely used in equine anaesthesia including as a part of PIVA. They are potent sedatives with good analgesic properties and also have side effects after bolus administration such as bradycardia, arrhythmias, decreases in cardiac output, and increases in systemic vascular resistance, respiratory depression, decreasing intestinal motility, and ataxia. The use of IV alpha-2 agonists in balanced anaesthetic techniques has become more and more popular, mainly to reduce MAC of volatile agents and to improve recovery during the perioperative period without significant cardiorespiratory effects.

Xylazine, the least selective alpha-2 agonist (selectivity ratio $\alpha 2: \alpha 1=160: 1$ ) has been shown to reduce MAC of isoflurane by $25 \%$ and $34 \%$ following IV administration of 0.5 and $1 \mathrm{mg} \cdot \mathrm{kg}^{-1}$ respectively [6]. Pöpel et al. [7] reported that xylazine applied as a CRI at rate $1 \mathrm{mg} \cdot \mathrm{kg}^{-1} \cdot \mathrm{hour}^{-1}$ after bolus of $0.6 \mathrm{mg} \cdot \mathrm{kg}^{-1}$ in isoflurane anaesthetized horses led to drop in anesthetic requirements and less need for blood pressure support compared to isoflurane alone.

A CRI of detomidine $(\alpha 2: \alpha 1=260: 1)$ at $5 \mu \mathrm{g} \cdot \mathrm{kg}^{-1} \cdot$ hour $^{-1}$ after bolus of $10 \mu \mathrm{g} \cdot \mathrm{kg}^{-1}$ administered in isoflurane anesthetized horses undergoing to elective surgeries demonstrated typical cardiovascular effects without any beneficial influence on isoflurane requirements, recovery duration and quality [8].

The use of romifidine ( $\alpha 2: \alpha 1=340: 1)$ as a CRI in isoflurane anesthetized horses submitted to elective surgeries was also described with contradictory results. According to Kuhn et al. [9] a CRI of $18 \mu \mathrm{g} \cdot \mathrm{kg}^{-1} \cdot \mathrm{hour}^{-1} \mathrm{after}$ initial dose of $80 \mu g \cdot \mathrm{kg}^{-1}$ caused a significant reduction in concentration of inhaled agent with improved cardiovascural and respiratory parameters. In contrast, Devisscher et al. [10] failed to detect neither inhalation sparing effect nor better cardiopulmonary function and recovery quality, when using the same loading dose of romifidine followed by a CRI at $40 \mu \mathrm{g} \cdot \mathrm{kg}^{-1} \cdot \mathrm{hour}^{-1}$.

Medetomidine is an extremely selective alpha-2 agonist $(\alpha 2: \alpha 1=1620: 1)$ and lower side effects could be assumed. PIVA using isoflurane and CRI of medetomidine has been studied widely in the equine anaesthesia. The application of $7 \mu \mathrm{g} \cdot \mathrm{kg}^{-1}$ IV followed by a CRI at $3.5 \mu \mathrm{g} \cdot \mathrm{kg}^{-1} \cdot \mathrm{hour}^{-1}$ in addition to isoflurane anaesthesia during orthopaedicsurgeries was found to reduce EtIso by $20 \%$ with lower dobutamine requirements to maintain arterial blood pressure and no differences in respiratory parameters comparatively to volatile anaesthetic alone [11].

Dexmedetomidine, the active enantiomer of medetomidine, is the most selective alpha-2 agonist $(\alpha 2: \alpha 1=$ 3240:1). It possesses beneficial pharmacokinetic properties such as short half-life and rapid distribution which render its use as a CRI very appropriate [12]. Administration of dexmedetomidine as an adjunctive agent to isoflurane anaesthesia produced an anaesthetic-sparing effects in human [13] [14] and dogs [15]. Its effects were studied on isoflurane anaesthetized horses by Marcilla et al. [16] [17]. In the former investigation the authors found out that the two CRIs used ( 1 and $1.75 \mu \mathrm{g} \cdot \mathrm{kg}^{-1} \cdot \mathrm{hour}^{-1}$ ) produced similar, small cardiopulmonary effects within an acceptable clinical range; in the former study they claimed that dexmedetomidine CRI at 1.75 $\mu \mathrm{g} \cdot \mathrm{kg}^{-1} \cdot$ hour $^{-1}$ after a bolus of $3.5 \mu \mathrm{g} \cdot \mathrm{kg}^{-1}$ did not reduce EtIso but improved recovery quality.

In the present study, we tested the effects of dexmedetomidine CRI on isoflurane requirements for maintenance of surgical anaesthesia as well as its influence on cardiopulmonary function, haemocoagulation and recovery in clinically healthy horses.

\section{Material and Methods}

\subsection{Animals}

The study was approved by the Ethical Committee of the Faculty of Veterinary Medicine at Trakia University in 
Stara Zagora.

Six healthy horses from local primitive Karakachan breed with average age 9.0 \pm 5.1 year and mean $( \pm$ SD) body weight $247.7 \pm 71.4 \mathrm{~kg}$ were included in the trial. Animals were bought from owners who intended to send them to slaughter house. They were kept in identical living and feeding conditions for a month before starting the experiment in order to eliminate the effect of environmental stress on the invest had variables. During this period a routine anthelmintic treatment was given using fenbendazole (Panacur ${ }^{\circledR}$, Intervet) at a dose of $7.5 \mathrm{mg} \cdot \mathrm{kg}^{-1}$ PO or mebendazol (Telmin ${ }^{\circledR}$, Janssen Pharmaceutical, Belgium) at the same dose. Clinical and laboratory investigations were also preliminarily performed and all animals were allocated in ASA grade I or II thereafter. Horses were assigned first to control/saline (ISO) group and next (two weeks apart) to experimental/dexmedetomidine (ISOD) group in order to produce cross-over design. Food but not water was deprived 12 hours before general anaesthesia.

\subsection{Experimental Design}

Experimental design was similar to our previously accomplished experiment using PIVA with halothane and dexmedetomidine CRI in horses [18].

Acepromazine maleate (Neurotranq ${ }^{\circledR}$, Alfasan International, Holland) was given IV at dose $0.03 \mathrm{mg} \cdot \mathrm{kg}^{-1} \mathrm{as} \mathrm{a}^{-1}$ premedication agent in the two groups. Xylazine hydrochloride (Alfasan International, Holland) $0.8 \mathrm{mg} \cdot \mathrm{kg}^{-1}$ was administered IV thirty minutes later through one of the two 14-gauge $2.1 \times 50 \mathrm{~mm}$ catheters (Venocan plus $^{\circledR}$, Kruuse, Denmark) placed in both jugular veins.

Anaesthesia was induced five to ten minutes after xylazine injection by mixture of ketamine hydrochloride (Anaket ${ }^{\circledR}$, Richter Pharma, Austria) $2.2 \mathrm{mg} \cdot \mathrm{kg}^{-1}$ with diazepam (Diazepam, Sopharma, Bulgaria) $0.05 \mathrm{mg} \cdot \mathrm{kg}^{-1}$ injected intravenously in the two protocols. Trachea was intubated with 20 - $22 \mathrm{~mm}$ OD tracheal tube (Cook) and the horses were moved to operation theatre where were placed on a padded surgical table in dorsal recumbency.

Anaesthesia was maintained for 3 hours with isoflurane (Foran ${ }^{\circledR}$, Abbot labretories, Switzerland) in oxygen $100 \%$ through closed circuit system of a large animal anaesthesia machine LDS 300 (Surgivet, USA) equipped with large animal ventilator DHV 1000 (Surgivet, USA) and out-of-circuit isoflurane vaporizer (Penion Limited Abington, Oxon, Ox $143 \mathrm{PH}, \mathrm{UK}$ ). As soon as the vaporizer was switched on, group ISOD received a CRI of dexmedetomidine hydrochloride (Dexdomitor ${ }^{\circledR}$, Orion Pharma, Finland) $1.75 \mu \mathrm{g} \cdot \mathrm{kg}^{-1} \cdot \mathrm{hour}^{-1}$ diluted with saline to a concentration of $0.01 \mathrm{mg} \cdot \mathrm{ml}^{-1}$, while group ISO received a CRI of equivalent volume of saline solution administered by means of microinfusion pump WZ-50C6 (All Pro, China) until the end of anaesthesia. Syringes were prepared in advance so as the anaesthetist was unaware of medications given.

Monitoring was performed throughout anaesthesia using a patient monitor PM-9000Vet (Mindray, China). The main clinical parameters heart rate (HR), electrocardiogram (ECG), haemoglobine oxygen saturation (Sat), respiratory rate (RR), inspired (FiIso) and expired (EtIso) fractions of isoflurane, inspired and expired fractions of $\mathrm{CO}_{2}\left(\mathrm{FiCO}_{2}, \mathrm{EtCO}_{2}\right)$, of $\mathrm{O}_{2}\left(\mathrm{FiO}_{2}, \mathrm{EtO}_{2}\right)$, and minimal alveolar concentration (MAC) of isoflurane were recorded every five minutes. For ECG recording II lead was used derived from sternal-wither configuration. Saturation probe was placed on the tongue.

Systolic (SYS), diastolic (DIA), and mean (MEAN) arterial blood pressures were measured invasively after cannulation of left or right facial artery using 22 -gauge $0.9 \times 25 \mathrm{~mm}$ catheter (Venocan plus ${ }^{\circledR}$, Kruuse, Denmark). Arterial blood samples were collected immediately after catheter placement and before the end of anaesthesia for blood gases, electrolytes and acid-base status measurement. Repiratory/blood gases VetStat ${ }^{\circledR}$ cassettes and VetStat ${ }^{\circledR}$ electrolyte and blood gas analyzer (IDEXX Laboratories, Inc., USA) were used for that purpose. Arterial lactate levels were measured by colorimetric method using enzymatic Roche/Hitachi lactate reagent (Roche Diagnostica, Germany).

Venous blood samples were taken at the beginning and at the end of anaesthesia in vials containing sodium citrate for measurement of some parameters of coagulation system such as fibrinogen, prothrombin time (PT), activated partial thromboplastin time (APTT), thrombin time (TT) using coagulometer Amelung KC1A (Germany) and tests of Human Diagnostica (Germany). Blood D-dimer was measured by means of quantitative latex-aglutination method (Spinreact, Spain).

Second venous catheter was used for administration of Ringer's solution (Actavis, Bulgaria) at a minimal rate of $10 \mathrm{ml} \cdot \mathrm{kg}^{-1} \cdot$ hour $^{-1}$ with some corrections throughout the anaesthesia. The rate was adjusted to maintain mean arterial pressure above $60 \mathrm{mmHg}$. Nevertheless, if blood pressure continued to drop, dopamine hydrochloride (Warsaw Pharmaseutical Works Polfa SA, Poland) was infused starting with a rate of $0.5 \mu \mathrm{g} \cdot \mathrm{kg}^{-1} \cdot \mathrm{minute}^{-1}$ and 
adjusted as required.

The animals were allowed to breathe spontaneously. If the arterial partial pressure of $\mathrm{CO}_{2}\left(\mathrm{PaCO}_{2}\right)$ increased above $60 \mathrm{mmHg}$, the arterial partial pressure of $\mathrm{O}_{2}\left(\mathrm{PaO}_{2}\right)$ decreased bellow $100 \mathrm{mmHg}$, or RR was lower than 4 breaths minute ${ }^{-1}$ for more than 3 minute an intermittent positive pressure ventilation (IPPV) was provided. Tidal volume of $20 \mathrm{ml} \cdot \mathrm{kg}^{-1}$, peak inspiratory pressure (PIP) no more $30 \mathrm{~cm} \mathrm{H}_{2} \mathrm{O}$, RR 8 breaths minute ${ }^{-1}$, and inspiratory time 2.5 seconds were set in an assisted-controlled mode of respiration. Urinary catheter was placed as well.

Deep surgical plane of anaesthesia was maintained by altering the vaporizer setting and thus inspired isoflurane concentrations. The depth of anaesthesia was assessed observing RR, HR, blood pressures, ocular position, movements, reflexes and moisture. Moreover, nociceptive electrical stimulation was given 30 minutes after induction of anaesthesia and every 30 minutes thereafter. For that purpose two surface electrodes were applied to the shaved and moistured skin over the lateral palmar digital nerve between the coronary band and the fetlock joint of the right hind limb, $1 \mathrm{~cm}$ apart and secured with elastic bandage. A constant current (CC) electrical stimulation was delivered [19] with the aim of an electrical stimulator Tonus 2M (Drujba Ltd., Vratza, Bulgaria). In case of positive reaction to electrical stimulation (gross purposeful movement of non stimulated limbs) or spontaneous movement of the horse without stimulation the isoflurane concentration was increased.

Three hours after tracheal intubation, the vaporizer was switched off and the animals allowed to recover in a quiet padded box. Horses were extubated as soon as they were able to swallow. No any assistance, oxygen supplementation, or additional sedation was applied during this period. The quality of recovery was evaluated by recording extubation time, time to sternal and time to standing positions, and observing the number of attempts to stand, the presence of violence, ataxia, or injury. The quality of recovery was scored from 1 to 5 grades (Table 1).

\subsection{Statistical Analysis}

Data were analyzed by means of a commercially available software package (Statistica ${ }^{\circledR}$ 6-0 version, StatSoft Inc. USA). The distribution of continuous data was tested using the Kolmogorov-Smirnov test. Analysis of variance (ANOVA) for repeated measurements was used to detect the influence of time and treatment upon each anaesthesiological or clinical variable. Factorial ANOVA was used to test the alterations in blood gases, electrolytes, and haemocoagulation parameters. Fisher LSD post-hoc analysis was performed in order to determine the probability value. Recovery durations were compared between two anaesthetic protocols using one-way ANOVA and post-hoc Fisher test. Recovery scores were compared in the Wilcoxon signed rank test. The minimal level of statistical significance was set at 0.05 for all analyses.

\section{Results}

HR, RR, Sat, SYS, MEAN, and DIA remained unchanged throughout isoflurane anaesthesia alone, whereas RR and Sat decreased over time in ISOD group with statistically significant alterations at 150 and 180 minutes comparatively to the initial period. Comparative analysis between groups showed several periods with lower HR, higher RR, SYS and MEAN values in horses anesthetized with PIVA using dexmedetomidine and isoflurane in relation to isoflurane anaesthesia alone (Table 2). The absence of changes in the ECG was found in both anaesthesia types for the entire period tracked.

The inspired and expired fractions of oxygen remained high during the whole anaesthesia in two groups (Table 3). $\mathrm{EtCO}_{2}$ was maintained in acceptable limits. The need for inhalation anaesthetic decreased over time assessed by Vol\%, MAC, FiIso, and EtIso. Anaesthetic requirements were lower in ISOD protocol which was

Table 1. Assessment of recovery quality in anaesthetized horses.

\begin{tabular}{c} 
Description \\
Getting up after one attempt, without ataxia \\
Getting up after one or two attempts, mild ataxia \\
More than two attempts to get up, but quiet \\
More than two attempts to get up, agitation \\
Significant violence, self-injury \\
4 \\
\hline
\end{tabular}




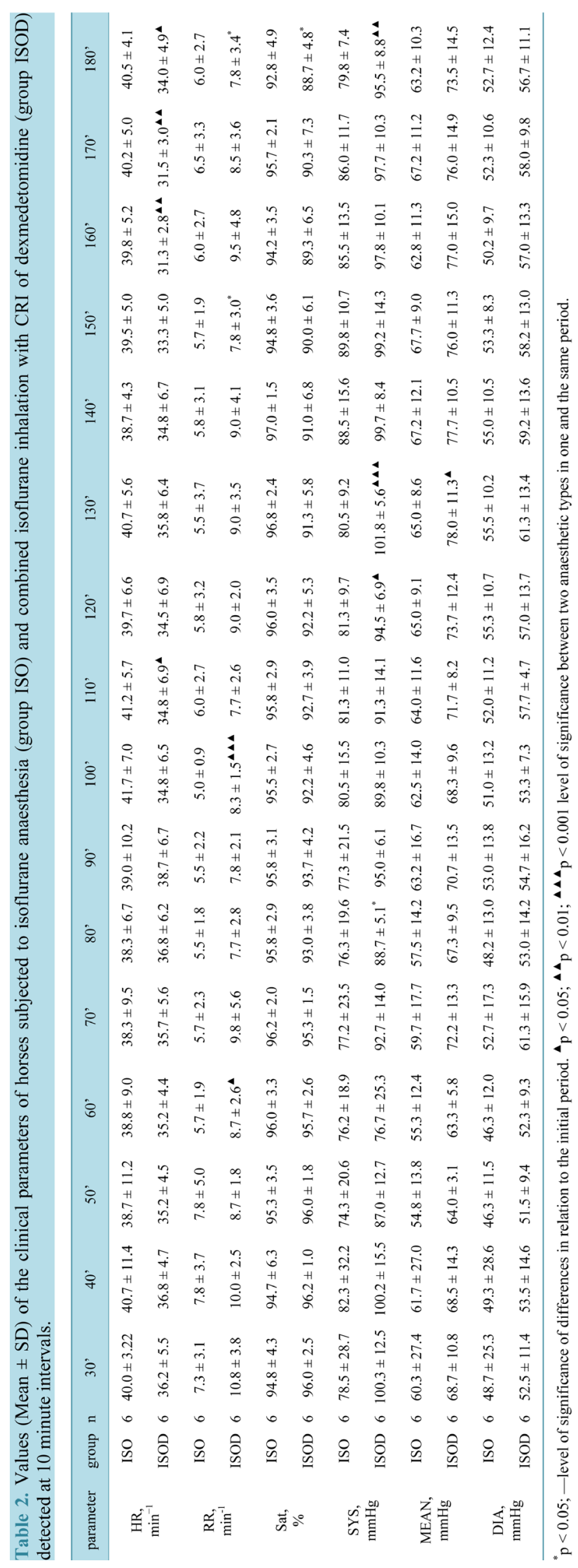




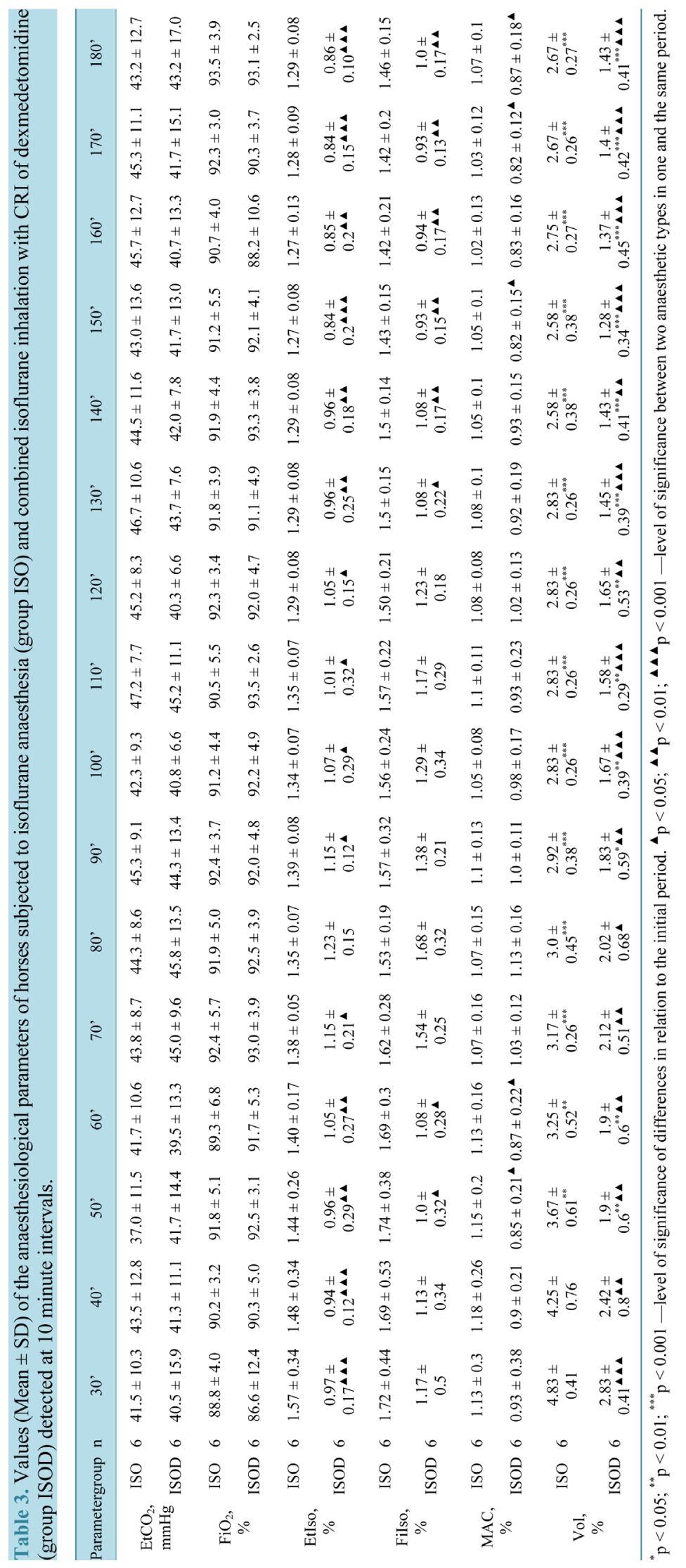


more pronounced at final periods. The mean reduction of MAC was calculated to be $15.6 \%$, EtIso was decreased by $23 \%$ in relation to ISO group. The drop in Vol\% was even more pronounced.

Both isoflurane and isoflurane-dexmedetomidine anaesthesias produced insignificant respiratory acidosis after 3 hours duration (Table 4). $\mathrm{PaO}_{2}$ significantly decreased in ISOD group comparatively to the initial values. At final period blood lactate levels increased in iso-dex anaesthesia in relation to the 0 period as well as to the isoflurane anaesthesia alone.

Coagulation parameters did not changed during both anaesthesia types with the exception of slightly higher fibrinogen levels in ISOD relatively to the ISO group at $3^{\text {rd }}$ hour (Table 5).

In terms of times and quality of recovery we did not find significant differences between the two groups (Table 6).

\section{Discussion}

According to the results of Marcilla et al. [16] aconstant rate infusion of dexmedetomidine produced similar cardiovascular effects in isoflurane anaesthetized horses independent of infusion rates used (1 and 1.75 $\mu \mathrm{g} \cdot \mathrm{kg}^{-1} \cdot \operatorname{hour}^{-1}$ ). We used the second rate as it is considered equipotent to CRI of the other alpha-2 agonists described in the introduction section [20]. This rate of dexmedetomidine used in our study renders enough to

Table 4. Blood gases and acid-base (BG-AB) parameters of horses (Mean \pm SD) subjected to isoflurane anaesthesia (group ISO) and combined isoflurane inhalation with CRI of dexmedetomidine (group ISOD).

\begin{tabular}{|c|c|c|c|c|}
\hline \multirow{2}{*}{ Parameters } & \multicolumn{2}{|c|}{ group ISO $(n=6)$} & \multicolumn{2}{|c|}{ group ISOD $(\mathrm{n}=6)$} \\
\hline & 0 Hour & 3 Hour & 0 Hour & 3 Hour \\
\hline $\mathrm{pH}$ & $7.418 \pm 0.07$ & $7.317 \pm 0.117$ & $7.428 \pm 0.054$ & $7.315 \pm 0.06$ \\
\hline $\mathrm{PaCO}_{2}, \mathrm{mmHg}$ & $50.0 \pm 12.47$ & $71.33 \pm 19.37$ & $45.0 \pm 6.2$ & $63.83 \pm 20.55$ \\
\hline $\mathrm{HCO}_{3}, \mathrm{mmol} / \mathrm{l}$ & $29.18 \pm 4.53$ & $32.68 \pm 2.25$ & $27.20 \pm 1.87$ & $29.23 \pm 4.8$ \\
\hline $\mathrm{tCO}_{2}, \mathrm{mmol} / \mathrm{l}$ & $30.70 \pm 4.79$ & $34.85 \pm 2.67$ & $28.6 \pm 1.96$ & $31.2 \pm 5.39$ \\
\hline $\mathrm{BE}, \mathrm{mmol} / \mathrm{l}$ & $4.33 \pm 3.37$ & $5.13 \pm 1.53$ & $2.87 \pm 1.96$ & $1.75 \pm 3.24^{\Perp}$ \\
\hline $\mathrm{PaO}_{2}$, mmHg & $175.33 \pm 59.17$ & $134.5 \pm 51.07$ & $187.17 \pm 34.25$ & $92.33 \pm 26.16^{* * *}$ \\
\hline $\mathrm{tHb}, \mathrm{g} / \mathrm{l}$ & $85.33 \pm 12.45$ & $80.17 \pm 17.24$ & $97.17 \pm 27.18$ & $107.5 \pm 37.0$ \\
\hline Sat, \% & $98.83 \pm 1.47$ & $97.17 \pm 2.32$ & $99.0 \pm 0.89$ & $93.17 \pm 4.79$ \\
\hline $\mathrm{Na}^{+}, \mathrm{mmol} / \mathrm{l}$ & $143.83 \pm 4.58$ & $144.17 \pm 3.06$ & $140.67 \pm 3.5$ & $142.0 \pm 7.59$ \\
\hline $\mathrm{K}^{+}, \mathrm{mmol} / \mathrm{l}$ & $4.17 \pm 0.8$ & $3.68 \pm 0.47$ & $4.48 \pm 0.77$ & $3.53 \pm 0.51$ \\
\hline $\mathrm{Cl}^{-}, \mathrm{mmol} / \mathrm{l}$ & $107.0 \pm 2.53$ & $106.67 \pm 2.5$ & $106.33 \pm 1.97$ & $105.17 \pm 6.68$ \\
\hline Lactate, $\mathrm{mmol} / \mathrm{l}$ & $1.16 \pm 0.49$ & $1.52 \pm 0.64$ & $1.64 \pm 0.86$ & $3.03 \pm 1.23^{* \boldsymbol{\Delta}}$ \\
\hline $\mathrm{AG}, \mathrm{mmol} / \mathrm{l}$ & $11.97 \pm 3.16$ & $8.45 \pm 1.07$ & $11.63 \pm 1.95$ & $11.0 \pm 3.25$ \\
\hline
\end{tabular}

${ }^{*} \mathrm{p}<0.05 ;{ }^{* * *} \mathrm{p}<0.001$ - level of significance of differences between two periods. ${ }^{\boldsymbol{\Delta}} \mathrm{p}<0.05$-level of significance of differences between two anaesthesia types for the respective period.

Table 5. Blood coagulation parameters of horses (Mean \pm SD) submitted to isoflurane anaesthesia (group ISO) and combined isoflurane inhalation with CRI of dexmedetomidine (group ISOD).

\begin{tabular}{cccc}
\hline & \multicolumn{2}{c}{ Group ISO $(\mathrm{n}=6)$} & Group ISOD (n=6) \\
\cline { 2 - 4 } Parameters & 0 hour & 3 hour & 0 hour \\
Фибриноген, g/l & $2.85 \pm 0.80$ & $2.34 \pm 0.75$ & $3.67 \pm 0.28$ \\
APTT, s & $53.05 \pm 10.23$ & $54.83 \pm 11.68$ & $57.68 \pm 10.38$ \\
PT, s & $11.78 \pm 0.84$ & $13.3 \pm 1.86$ & $14.42 \pm 1.31$ \\
TT, s & $18.70 \pm 2.53$ & $20.17 \pm 1.66$ & $18.93 \pm 3.43$ \\
D-dimer, $\mu \mathrm{g} / \mathrm{ml}$ & $1.40 \pm 0.62$ & $1.86 \pm 0.99$ & $0.97 \pm 0.55$ \\
\hline
\end{tabular}

$\Delta \mathrm{p}<0.05$; level of significance of differences between two anaesthesia types for the respective period. 
Table 6. Recovery score and times in isoflurane anaesthetized horses with (group ISOD) and without (group ISO) a CRI of dexmedetomidine.

\begin{tabular}{ccc}
\hline Parameters & Group ISO $(\mathrm{n}=6)$ & Group ISOD $(\mathrm{n}=6)$ \\
\hline Score (Mean \pm SD) & $2.17 \pm 0.75$ & $1.67 \pm 0.52$ \\
Score Median (IQR) & $2(2-3)$ & $2(1-2)$ \\
Time of extubation, min. & $8.0 \pm 2.45$ & $7.33 \pm 2.58$ \\
Time for sternal recumbancy, min. & $18.5 \pm 8.26$ & $21.0 \pm 4.34$ \\
Time for standing, min. & $41.17 \pm 9.15$ & $38.33 \pm 6.15$ \\
\hline
\end{tabular}

reduce isoflurane requirements for maintenance of surgical anaesthesia, but the degree of reduction was lower than expected. The reason might be the lack of loading dose of dexmedetomidine in our protocol where it was replaced by xylazine premedication. Another possible cause could be the low CRI used to supplement the inhalation agent. The way of calculation of MAC reduction was not uniform in all investigations which might result in different percentage of decrease in volatile agent concentration during PIVA. The drop in isoflurane needs was much more pronounced when PIVA was combined with CRIs of other agents such as lidocaine, midazolam, or ketamine [21] [22]. Lidocaine proved to decrease MAC of volatile agents dose-dependantly but leads to ataxia during recovery period. Ketamine also has potentiated inhalation anaesthesia but the occurrence of excitation during recovery could not be prevented. Therefore, alpha-2 agonists remained the agents of choice for PIVA.

We observed stable cardiovascular function during both isoflurane and isoflurane-dexmedetomidine protocols with several periods of elevated systolic and mean arterial pressures in ISOD group. This difference probably was connected with the effect of dopamine infusion to maintain blood pressures above safe limits. Horses anaesthetized with isoflurane-dexmedetomidine received the total of 35 mililitres, whereas horses from ISO group were given 30 mililiters of dopamine. HR was lower compared to ISO group, a typical side effect of alpha-2 agonists that was reported by other authors [17] [21].

Although all animals were breathing $100 \%$ of oxygen and the lowest inspired and expired fractions of oxygen were $86.6 \%$ and $79.0 \%$, respectively, we found out gradually drop in $\mathrm{PaO}_{2}$ that was more obvious in ISOD group. This effect has been reported by other investigators [17] and resulted in elevated lactate levels. The respiratory function in horses is altered by anaesthetic drugs and bodyposition especially during dorsal recumbency. A reduction of $\mathrm{PaO}_{2}$ is caused by diffusion abnormalities, ventilation-perfusion mismatching or right to left shunts independent on 50\% or maximal inspired oxygen concentration [23], whereas $\mathrm{PaCO}_{2}$ is rarely elevated under these conditions because of the stimulation of ventilation as a result of hypoxaemia [24]. The $\mathrm{EtCO}_{2}$ and $\mathrm{PaCO}_{2}$ correlate positively but in horses $\mathrm{EtCO}_{2}$ tends to be 10 to $15 \mathrm{mmHg}$ lower than $\mathrm{PaCO}_{2}$ [25]. IPPV was necessitated in one horse from ISO group and three horses in ISOD group. Hypoventilation is a common finding during equine anaesthesia because virtually all drugs cause respiratory depression. The increase in $\mathrm{PaCO}_{2}$ during isoflurane anesthesia is attributable to a decrease in respiratory rate, whereas tidal volume is maintained or increases [26]. Horses anesthetized with isoflurane in 100\% oxygen often have an irregular ventilatory rhythm, characterized by a low respiratoryrate. The addition of dexmedetomidine CRI to isoflurane increased RR but did not improved ventilation and oxygenation.

Changes in coagulation system during general anaesthesia in horses were rarely investigated. Inhalation anaesthetics inhibit thrombocyte aggregation at MAC above 1 . However, this effect did not result in alteration of measured coagulation parameters. Our results are in accordance with the study of Elrashidy et al. [27] where isoflurane did not have any effect either on thrombocyte aggregation or on the rest of coagulation parameters. The difference in fibrinogen values between groups at $3^{\text {rd }}$ hour resulted from slight decrease in isoflurane anaesthesia.

For equine anaesthesia, $25 \%$ - 50\% of fatalities are a direct result of injury sustained during recovery [1] [28]. In addition to the horse's physical condition and temperament, the environment at the recovery site and the type of surgery, the quality and time of recovery is related to the dose and route of anaesthetic drug administration, the duration of anaesthesia, the cardiopulmonary function during maintenance of anaesthesia and the administration of sedatives or drug antagonists during the recovery period [1]. A challenge for the anaesthetist is to find a dosing regimen that provides adequate anaesthetic depth and ensures that horses regain sufficient strength and coordination before they try to stand up. Our experimental horses showed good quality of recovery with similar scores for both isoflurane anaesthesia alone and isoflurane-dexmedetomidine without any sedation, oxygen sup- 
plementation, or assistance. Times for extubation, for sternal and standing positions also were similar between groups. Therefore, dexmedetomidine CRI did not influence the time and quality of recovery during isoflurane anaesthesia in healthy horses which was opposite to the data of Marcilla et al. [17] who reported better but longer recovery when dexmedetomidine was added to isoflurane.

\section{Conclusion}

In conclusion, the use of dexmedetomidine CRI as an anesthetic adjunct to isoflurane anaesthesia reduced slightly isoflurane requirements, maintained stable cardiovascular function, produced greater respiratory depression than isoflurane alone, and had no effects on haemocoagulation as did isoflurane alone. Recovery times and quality were good and comparable between groups.

\section{References}

[1] Johnston, G., Eastment, J., Wood, J. and Taylor, P. (2002) The Confidential Enquiry into Postoperative Equine Fatalities (CEPEF): Mortality Results of Phases 1 and 2. Veterinary Anaesthesia and Analgesia, 29, 159-170. http://dx.doi.org/10.1046/j.1467-2995.2002.00106.x

[2] Johnston, G., Eastment, J., Taylor, P. and Wood, J. (2004) Is Isoflurane Safer than Halothane in Equine Anaesthesia? Result from a Prospective Multicentre Randomized Controlled Trial. Equine Veterinary Journal, 36, 64-71. http://dx.doi.org/10.2746/0425164044864723

[3] Brosnan, R.J. (2013) Inhaled Anesthetics in Horses. Veterinary Clinics of North America: Equine Practice, $29,69-87$. http://dx.doi.org/10.1016/j.cveq.2012.11.006

[4] Gonzalo-Marcilla, M., Gasthuys, F. and Schauvliege, S. (2014) Partial Intravenous Anaesthesia in the Horse: A Review of Intravenous Agents Used to Supplement Equine Inhalation Anaesthesia. Part 1: Lidocaine and Ketamine. Veterinary Anaesthesia and Analgesia, 41, 335-345. http://dx.doi.org/10.1111/vaa.12179

[5] Gonzalo-Marcilla, M., Gasthuys, F. and Schauvliege, S. (2015) Partial Intravenous Anaesthesia in the Horse: A Review of Intravenous Agents Used to Supplement Equine Inhalation Anaesthesia. Part 2: Opioids and Alpha-2 Adrenoceptor Agonists. Veterinary Anaesthesia and Analgesia, 42, 1-16. http://dx.doi.org/10.1111/vaa.12196

[6] Steffey, E.P., Pascoe, P.J., Woliner, M.J. and Berryman, E.R. (2000) Effects of Xylazine Hydrochloride during Isoflurane-Induced Anesthesia in Horses. American Journal of Veterinary Research, 61, 1225-1231. http://dx.doi.org/10.2460/ajvr.2000.61.1225

[7] Pöppel, N.F., Hopster, K., Geburek, F. and Kästner, S. (2015) Influence of Ketamine or Xylazine Supplementation on Isoflurane Anaesthetized Horses-A Controlled Clinical Trial. Veterinary Anaesthesia and Analgesia, 42, 30-38. http://dx.doi.org/10.1111/vaa.12176

[8] Schauvliege, S., Marcilla, M.G., Verryken, K., Duchateau, L., Devisscher, L. and Gasthuys, F. (2011) Effects of a Constant Rate Infusion of Detomidine on Cardiovascular Function, Isoflurane Requirements and Recovery Quality in Horses. Veterinary Anaesthesia and Analgesia, 38, 544-554. http://dx.doi.org/10.1111/j.1467-2995.2011.00659.x

[9] Kuhn, M., Köhler, L., Fenner, A., Enderle, A. and Kampmann, C. (2004) Isoflurane Sparing and the Influence on Cardiovascular and Pulmonary Parameters through a Continuous Romifidine Hydrochloride Infusion during General Anaesthesia in Horses-A Clinical Study. Pferdeheilkunde, 20, 511-516.

[10] Devisscher, L., Schauvliege, S., Dewulf, J. and Gasthuys, F. (2010) Romifidine as a Constant Rate Infusion in Isoflurane Anaesthetized Horses: A Clinical Study. Veterinary Anaesthesia and Analgesia, 37, 425-433. http://dx.doi.org/10.1111/j.1467-2995.2010.00556.x

[11] Neges, K., Bettschart-Wolfensberger, R., Müller, J., Fürst, A. and Kästner, S. (2003) The Isoflurane Sparing Effect of a Medetomidine Constantrate Infusion in Horses. Veterinary Anaesthesia and Analgesia, 30, 92-93. http://dx.doi.org/10.1046/j.1467-2995.2003.00132_8.x

[12] Bettschart-Wolfensberger, R., Freeman, S.L., Bowen, I.M., Aliabadi, F.S., Weller, R., Huhtinen, M. and Clarke, K.W. (2005) Cardiopulmonary Effects and Pharmacokinetics of i.v. Dexmedetomidine in Ponies. Equine Veterinary Journal, 37, 60-64. http://dx.doi.org/10.2746/0425164054406801

[13] Aanta, R., Jaakola, M., Kallio, A. and Kanto, J. (1997) Reduction of the Minimun Alveolar Concentration of Isoflurane by Dexemedetomidine. Anesthesiology, 86, 1055-1060. http://dx.doi.org/10.1097/00000542-199705000-00008

[14] Khan, Z.P., Munday, I.T., Jones, R.M., Thornton, C., Mant, T.G. and Amit, D. (1999) Effects of Dexmedetomidine on Isoflurane Requirements in Healthy Volunteers, 1: Pharmacodynamic and Pharmacokinetic Interactions. British Journal of Anaesthesia, 83, 372-380. http://dx.doi.org/10.1093/bja/83.3.372

[15] Pascoe, P.J., Raekallio, M., Kuusela, E., McKusick, B. and Granholm, M. (2006) Changes in the Minimum Alveolar 
Concentration of Isoflurane and Some Cardiopulmonary Measurements during Three Continuous Infusion Rates of Dexmedetomidine in Dogs. Veterinary Anaesthesia and Analgesia, 33, 97-103. http://dx.doi.org/10.1111/j.1467-2995.2005.00236.x

[16] Marcilla, M.G., Schauvliege, D., Duchateau, L. and Gasthuys, F. (2010) Cardiopulmonary Effects of Two Constant Rate Infusions of Dexmedetomidine in Isoflurane Anaesthetizsd Ponies. Veterinary Anaesthesia and Analgesia, 37, 311-321. http://dx.doi.org/10.1111/j.1467-2995.2010.00537.x

[17] Marcilla, M.G., Schauvliege, S., Segaert, S., Duchateau, L. and Gasthuys, F. (2012) Influence of a Constant Rate Infusion of Dexmedetomidine on Cardiopulmonary Function and Recovery Quality in Isoflurane Anaesthetized Horses. Veterinary Anaesthesia and Analgesia, 39, 49-58. http://dx.doi.org/10.1111/j.1467-2995.2011.00672.x

[18] Simeonova, G. and Sleiman, M. (2014) Comparison of Effects of Halothane Anaesthesia in Horses with and without Dexmedetomidine Continuous Rate Infusion. Advances in Animal and Veterinary Sciences, 2, 418-423. http://dx.doi.org/10.14737/journal.aavs/2014/2.7.418.423

[19] Levionnois, O., Spadavecchia, C., Kronen, P. and Schatzmann, U. (2009) Determination of the Minimum Alveolar Concentration of Isoflurane in Shetland Ponies Using Constant Current or Constant Voltage Electrical Stimulation. Veterinary Anaesthesia and Analgesia, 36, 9-17. http://dx.doi.org/10.1111/j.1467-2995.2008.00420.x

[20] Mueller, C., Hopster, K., Hopster-Iversen, C. and Kaestner, S.B.R. (2012) Elaboration of a Xylazine and Dexmedetomidine Infusion Regime Which Provides a Constant Level of Sedation Inhorses. Pferdeheilkunde, 28, 668-674.

[21] Hopster, K., Müller, C., Hopster-Iversen, C., Stahl, J., Rohn, K. and Kästner, S. (2014) Effects of Dexmedetomidine and Xylazine on Cardiovascular Function during Total Intravenous Anaesthesia with Midazolam and Ketamine and Recovery Quality and Duration in Horses. Veterinary Anaesthesia and Analgesia, 41, 25-35. http://dx.doi.org/10.1111/vaa.12095

[22] Duke-Novakovski, T., Palacios-Jimenez, C., Wetzel, T., Rymes, L. and Sanchez-Teran, A.F. (2015) Cardiopulmonary Effects of Dexmedetomidine and Ketamine Infusions with Either Propofol Infusion or Isoflurane for Anesthesia in Horses. Veterinary Anaesthesia and Analgesia, 42, 39-49. http://dx.doi.org/10.1111/vaa.12194

[23] Hubbell, J.A.E., Aarnes, T.K., Bednarski, R.M., Lerche, P. and Muir, W.W. (2011) Effect of 50\% and Maximal Inspired Oxygen Concentrations on Respiratory Variables in Isoflurane-Anesthetized Horses. BMC Veterinary Research, 7, 23. http://www.biomedcentral.com/1746-6148/7/23 http://dx.doi.org/10.1186/1746-6148-7-23

[24] Hubbell, J.A.E. and Muir, W.W. (2009) Monitoring Anesthesia. In: Muir, W.W. and Hubbell, J.A., Eds., Equine Anesthesia Monitoring and Emergency Therapy, Saunders Elsevier, St. Louis, 149-170. http://dx.doi.org/10.1016/b978-1-4160-2326-5.00008-0

[25] Yamashita, K. and Muir, W.W. (2009) Intravenous Anesthetic and Analgesic Adjuncts to Inhalation Anesthesia. In: Muir, W.W. and Hubbell, J.A., Eds., Equine Anesthesia Monitoring and Emergency Therapy, Saunders Elsevier, St. Louis, 260-276. http://dx.doi.org/10.1016/b978-1-4160-2326-5.00013-4

[26] Crumley, M.N., McMurphy, R.M., Hodgson, D.S. and Kreider, S.E. (2013) Effects of Inspired Oxygen Concentration on Ventilation, Ventilatory Rhythm, and Gas Exchange in Isoflurane-Anesthetized Horses. American Journal of Veterinary Research, 74, 183-190. http://dx.doi.org/10.2460/ajvr.74.2.183

[27] Elrashidy, A.A., Abdelrahman, R.S., Ghali, A.M., Elsheikh, A.M. and Elsheikh, M.M. (2007) Effects of Sevoflurane and Isoflurane on Coagulation System: A Comparative Study. Tanta Medical Sciences Journal, 2, 11-21.

[28] Bidwell, L.A., Bramlage, L.R. and Rood, W.A. (2007) Equine Perioperative Fatalities Associated with General Anaesthesia at a Private Practice-A Retrospective Case Series. Veterinary Anaesthesia and Analgesia, 34, 23-30. http://dx.doi.org/10.1111/j.1467-2995.2005.00283.x 